\title{
Enhancement of Image Sharpness with Bilateral and Adaptive Filter
}

\author{
Ch. Ravi Kumar, Member, IACSIT and S. K. Srivatsa
}

\begin{abstract}
The two most common forms of degradation, an image suffers are loss of sharpness or blur, and noise. In this paper, an attempt has been made to first develop a sharpening method that increases the slope of edges without producing overshoot and undershoot, which renders clean, crisp, and artifact-free edges, thereby improving the overall appearance of the image. In this paper, we present the adaptive bilateral filter (ABF) for sharpness enhancement and noise removal. The ABF sharpens an image by increasing the slope of the edges without producing overshoot or undershoot. It is an approach to sharpness enhancement that is fundamentally different from the un sharp mask. This new approach to slope restoration also differs previous slope restoration algorithms. In the ABF, the edge slope is enhanced by transforming the histogram via a range filter with adaptive offset and width. The $A B F$ is able to smooth the noise, while enhancing edges and textures in the image. The parameters of the ABF are optimized with a training procedure.. The proposed method is effective at removing signal noise while enhancing the experimental results in perceptual quality both quantitatively and qualitatively.
\end{abstract}

Index Terms-Bilateral filter, de-blurring, noise removal, range filter, sharpness.

\section{INTRODUCTION}

Image restoration refers to the genre of techniques that aim to recover a high quality original image from a degraded version of that image given a specific model for degradation process [1]. Thus restoration techniques are oriented towards modeling the degradation and applying the inverse process in order to recover the original image. This is in contrast to image enhancement techniques that seek to improve the appearance of an image without reference to a specific model for the degradation process. The restoration framework is particularly valuable because in conjunction with a training-based approach, it provides a context within which the free parameters of the restoration algorithm may be optimized.

Training-based approaches have been used to develop imaging algorithms for a variety of applications, including image interpolation, image restoration, digital half toning, discerning, and color correction.

The ingredients that training-based approaches have in common when used for development of imaging algorithms are: 1) a set of training pairs each consisting of an input image and a desired output image, 2) an architecture for the

Manuscript received May 12, 2014; revised July 28, 2014.

Ch. Ravi Kumar is with Prakasam Engineering College, Kandukur, Prakasam (Dt) Andhra Pradesh, India (e-mail: ravi_ece99@yahoo.com).

S. K. Srivatsa is with the Department of ECE, Anna University, Chennai, India (e-mail: profsks@ @rediffmail.com). algorithm consisting of free parameters, and 3) a cost function under which those free parameters may be optimized. In many cases, the architecture contains a classifier that allows for parameter optimization separately within different pixel classes according to the value of an appropriately chosen feature vector. In this paper, we propose a new training-based approach to image restoration. Once the restoration algorithm has been fully developed, we are, however, free to apply it to images for which the degradation process is unknown. This puts us back in the domain of enhancement. The two most common forms of degradation an image suffers are loss of sharpness or blur, and noise. The degradation model we use consists of a linear, shift-invariant blur followed by additive noise, described in detail in [2]. The problem we are interested in is twofold. First we seek to develop a sharpening method that is fundamentally different from the un sharp mask filter (USM) which sharpens an image by enhancing the high-frequency components of the image. In the spatial domain, the boosted high-frequency components lead to overshoot and undershoot around edges, which causes objectionable ringing or halo artifacts. Our goal is to develop a sharpening algorithm that increases the slope of edges without producing overshoot and undershoot, which renders clean, crisp, and artifact-free edges, thereby improving the overall appearance of the image.

\section{BILATERAL Filter}

Traditional filtering [3] is domain filtering, and enforces closeness by weighing pixel values with coefficients that fall off with distance. Similarly, range filtering, averages image values with weights that decay with dissimilarity. In terms of noise removal, conventional linear filters work well for removing additive Gaussian noise, but they also significantly blur the edge structures of an image. Therefore, a great deal of research has been done on edge-preserving noise reduction. One of the major endeavors in this area has been to utilize rank order information. Due to a lack of the sense of spatial ordering, rank order filters generally do not retain the frequency selective properties of the linear filters and do not suppress Gaussian noise optimally Range filters are nonlinear because their weights depend on image intensity or color. Computationally, they are no more complex than standard non separable filters. Most importantly, they preserve edges. Spatial locality is still an essential notion.

In fact, it is shown that range filtering by itself merely distorts an image's color map. Then the combination of range and domain filtering is done, and shown that the combination is much more interesting. The combined filtering is known as 
bilateral filtering. Since bilateral filters assume an explicit notion of distance in the domain and in the range of the image function, they can be applied to any function for which these two distances can be defined. In particular, bilateral filters can be applied to color images just as easily as they are applied to black-and-white ones [4].

The CIE-Lab color space endows the space of colors with a perceptually meaningful measure of color similarity, in which short Euclidean distances correlate strongly with human color discrimination performance. Thus, if we use this metric in our bilateral filter, images are smoothed and edges are preserved in a way that is tuned to human performance. Only perceptually similar colors are averaged together, and only perceptually visible edges are preserved. The bilateral filter proposed by Tomasi and Manduchi in 1998 is a nonlinear filter that smoothes the noise while preserving edge structures. The shift-variant filtering operation of the bilateral filter is given by

$$
f[m, n]=\sum_{k} \sum_{l} h[m, n ; k, l] g[k, l]
$$

where $f[m, n]$ is the restored image, $h[m, n ; k, l]$ is the response at at $[m, n]$ to an impulse at $[k, l]$ and $g[m, n]$ is the degraded image.

For the bilateral filter, $\left[m_{0}, n_{0}\right]$ is the center pixel of the window,

$$
\Omega_{m_{0}, n_{0}}=\{[m, n]\}:
$$

$\left.[m, n] \in\left[m_{0}-N, m_{0}+N\right] \times\left[n_{0}-N, n_{0}+N\right]\right\}, \sigma_{d}, \sigma_{r}$. are the standard deviations of the domain and range Gaussian filters, respectively, and

$$
\begin{aligned}
r_{m_{0}, n_{0}}= & \sum_{m=m_{0}-N}^{m_{0}+N} \sum_{n=n_{0}-N}^{n_{0}+N} \exp \left(-\frac{\left(m-m_{0}\right)^{2}+\left(n-n_{0}\right)^{2}}{2 \sigma_{d}^{2}}\right) \\
& \times \exp \left(-\frac{\left(g[m, n]-g\left[m_{0}, n_{0}\right]\right)^{2}}{2 \sigma_{r}{ }^{2}}\right)
\end{aligned}
$$

is a normalization factor that assures that the filter preserves average gray value in constant areas of the image. The edge-preserving de-noising bilateral filter adopts a low pass Gaussian filter for both the domain filter and the range filter. The domain low-pass Gaussian filter gives higher weight to pixels that are spatially close to the center pixel. The range low pass Gaussian filter gives higher weight to pixels that are similar to the center pixel in gray value [5]. Combining the range filter and the domain filter, a bilateral filter at an edge pixel becomes a elongated Gaussian filter that is oriented along the edge. This ensures that averaging is done mostly along the edge and is greatly reduced in the gradient direction. This is the reason why the bilateral filter can smooth the noise while preserving edge structures [6]. Combined domain and range filtering will be denoted as bilateral filtering.

As a consequence, the bilateral filter acts essentially as a standard domain filter, and averages away the small, weakly correlated differences between pixel values caused by noise.

Fig. 1(b) shows that a bilateral filter with $\sigma_{d}=2$.and $\sigma_{r}=20$ removes much of the noise that appears in the degraded image shown in Fig. 1(a) and preserves the edge structures. In Fig. 1(c), where the spatial domain Gaussian with $\tau_{d}=2$ is applied alone, the edges are significantly blurred. The transfer function of the bilateral filter shown in Fig. 1(d) demonstrates that the bilateral filter at pixel A is low pass in one direction, and almost all-pass in the orthogonal direction. This explains, from a frequency domain perspective why this filter is able to preserve edges while removing noise. On the other hand, the bilateral filter is essentially a smoothing filter. It does not sharpen edges. The edge rendered by the bilateral filter has the same level of blurriness as in the original degraded image, although the noise is greatly reduced [7].

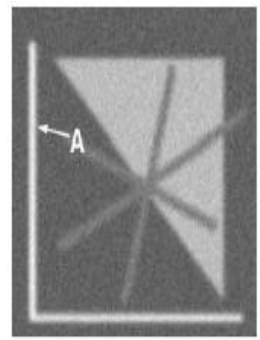

(a)

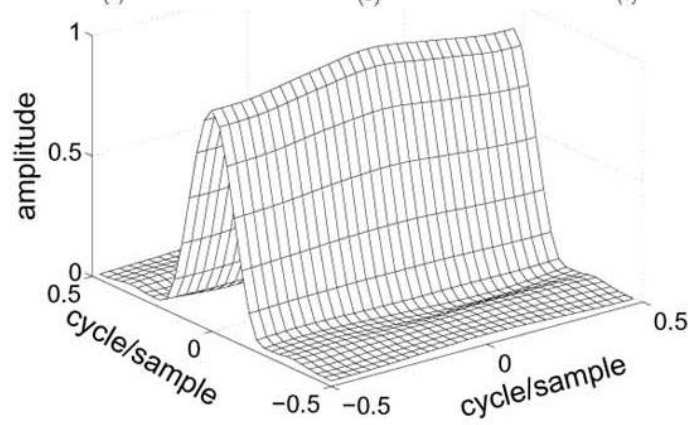

(d)

Fig. 1. Illustration of how the bilateral filter works. (a) Blurred and noisy 165_156 image "edge.tif"; (b) bilateral filter restored image $\left({ }_{-}=20 ;_{-}=2\right)$; (c) Gaussian filter restored image $(=2)$.

The results of the bilateral filtering are a significant improvement over a conventional linear low-pass filter. However, in order to enhance the sharpness of an image, we need to make some modifications to this filter.

\section{ADAPTIVE BILATERAL FILTER (ABF) FOR SHARPENING OF IMAGE}

The Adaptive Bilateral Filter is a new training based approach to image restoration. It also consists of two filters: 1 . Domain Filter; 2. Range Filter. ABF restored images are significantly sharper than those restored by the bilateral filter. $\mathrm{ABF}$ works well for both gray images and color images.

The domain filter is mainly used for noise removal. Domain filter gives higher weight to pixels that are spatially close to the center pixel. Range filter acts as a derivative filter that processes the histogram of the image for sharpness. The range filter gives higher weight to pixels that are similar to the center pixel in gray value. In this section, we present a new sharpening and smoothing algorithm: the adaptive 
bilateral filter $(\mathrm{ABF})$. The range filter can be interpreted as a 1-D filter that processes the histogram of the image.

The parameter $\sigma r$ of the range filter controls the width of the range filter. It determines how selective the range filter is in choosing the pixels that are similar enough in gray value to be included in the averaging operation. If is large compared to the range of the data in the window, the range filter will assign similar weight to every pixel in the range.

The response at $\left[m_{\mathrm{o}}, n_{\mathrm{o}}\right]$ of the proposed shift-variant ABF to an impulse at $[m, n]$ is given by (4), where $\left[m_{\mathrm{o}}, n_{\mathrm{o}}\right]$ and $\Omega_{m_{0}, n_{0}}$ are defined as before, and the normalization factor is given by

$$
\begin{gathered}
r_{m 0, n 0}=\sum_{m=m_{0}-N}^{m_{0}+N} \sum_{n=n_{0}-N}^{n_{0}+N} \exp \left(-\frac{\left(m-m_{0}\right)^{2}+\left(n-n_{0}\right)^{2}}{2 \sigma_{d}^{2}}\right) \\
\times \exp \left(\frac{\left(g[m, n]-g\left[m_{0}, n_{0}\right]-\varsigma\left[m_{0}, n_{o}\right]\right)^{2}}{2 \sigma_{r}{ }^{2}\left[m_{\cdot 0}, n_{0}\right]}\right. \\
h\left[m_{0}, n_{0}: m, n\right]=\{r-1 \\
\exp \left(\frac{\left(m-m_{0}\right)^{2}+\left(n-n_{0}\right)^{2}}{2 \sigma_{d}^{2}}\right) \\
\exp \left(-\frac{\left(g[m, n]-g\left[m_{0}, n_{0}\right]-\varsigma\left[m_{0}, n_{0}\right]\right\}^{2}}{2 \sigma r^{2}}\right), \\
{[m, n] \in \Omega m_{0}, n_{0}}
\end{gathered}
$$

The ABF retains the general form of a bilateral filter, but contains two important modifications. First, an offset $\checkmark$ is introduced to the range filter in the ABF. Second, both $\checkmark$ and the width of the range filter $\sigma_{r}$ in the ABF are locally adaptive. If $\varsigma=0$ and $\sigma_{r}$ is fixed, the ABF will degenerate into a conventional bilateral filter. For the domain filter, a fixed low-pass Gaussian filter with $\sigma_{d}=1.0$ is adopted in the ABF. The combination of a locally adaptive $\zeta$ and $\sigma_{r}$ transforms the bilateral filter into a much more powerful filter that is capable of both smoothing and sharpening. The parameter $\sigma_{r}$ of the range filter controls the width of the range filter. It determines how selective the range filter is in choosing the pixels that are similar enough in gray value to be included in the averaging operation. If $\sigma_{r}$ is large compared to the range of the data in the window, the range filter will assign similar weight to every pixel in the range. Then it will not have much effect on the overall bilateral filter. On the other hand a small $\sigma_{r}$ will make the range filter dominate the bilateral filter [8].

Moreover, it sharpens an image by increasing the slope of the edges. It determines how selective the range filter is in choosing the pixels that are similar enough in gray value to be included in the averaging operation.

\section{Feature Design}

The feature for pixel classification plays an important role in the success of the training. We described how shifting the range filter according to the difference between the center pixel value and the mean of the local data window will impact the output image. General guidelines for choosing the feature (s) are:

1) be able to reflect the strength of edges;

2) it can distinguish the regions, to process differently, mainly, the regions for smoothing and sharpening;

3) it have some robustness to noise.

Laplacian of Guassian (Log) Operator:

The Laplacian of Gaussian is a feature used for pixel classification. It is used to determine whether a pixel belongs to bright or dark side of an edge. It calculates Laplacian. It is a high pass filter and computes the second order derivative of input image. The magnitude of response near edges is high. In smooth regions [9] and the magnitude of response is low. On the center of edge, response is zero. Magnitude of LoG strength reflects the local edge structure. More robust to noise compared to other operators. The sign of response gives the location of a pixel with respect to edge.

\section{RESUlTS AND DisCUSSIONS:}

The bilateral filter has been widely used since it was first proposed. The ABF is an extension of the traditional bilateral filter. With its capability of adaptive sharpening and smoothing, it offers the potential for wider application and better image quality. Although the ABF is developed in the framework of image restoration, it can be used as an image enhancing algorithm, as we have demonstrated with the test images. In particular, our main motivation for developing the $\mathrm{ABF}$ is to enhance the quality of digital images that consumers want to view and/or print. The performance of the $\mathrm{ABF}$ is evaluated on gray scale image and is compared with bilateral filter.

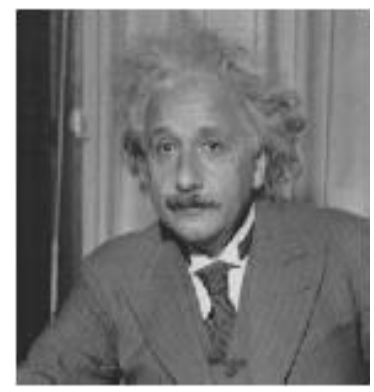

a)

Fig. 2 a). Input image; b). Degraded image.

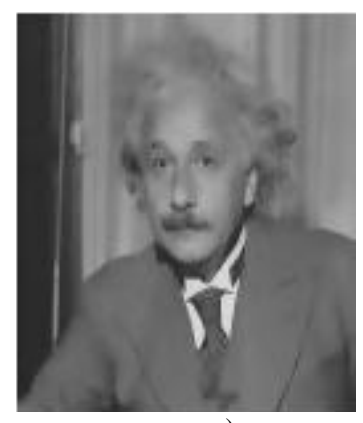

a)

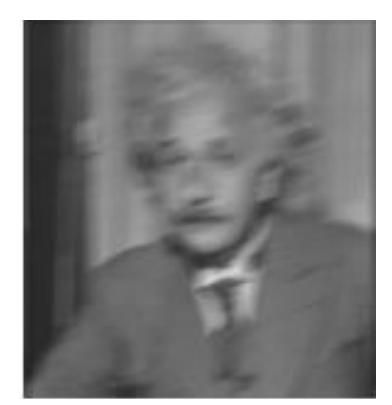

b).

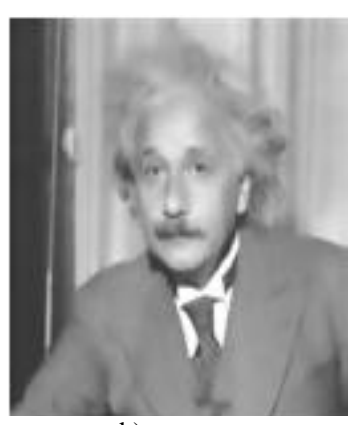

b).
Fig. 3 a). Bilateral filtered image; b). ABF filtered image.

Fig. 4(a) and 4(b) shows the effect of bilatera adaptive filter on colour images with different values of $\sigma_{r}$ 


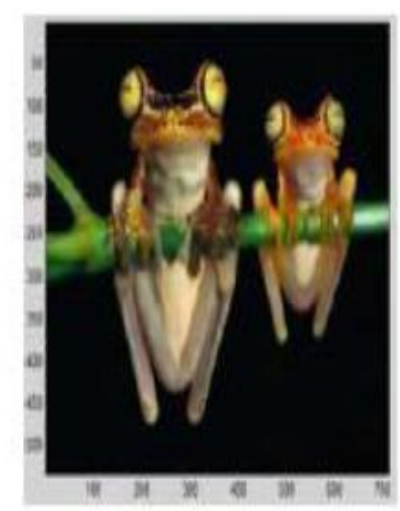

$\sigma_{r}=14$

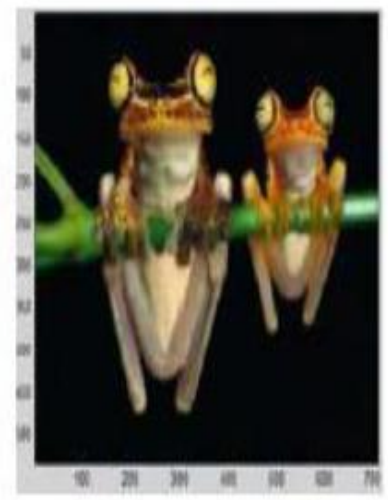

$\sigma_{r}=20$

a)

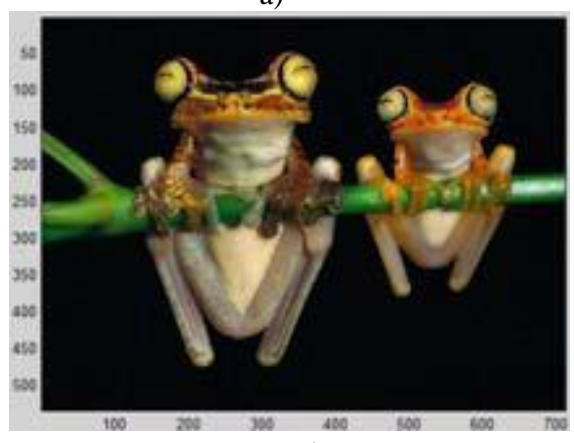

b) $\sigma_{r}=1$
Fig. 4. Effect of $\mathrm{ABF}$ on color image: Variance $(\sigma r)$ of adaptive bilateral filter: a) $\sigma_{r}=14$, b) $\sigma_{r}=1$.

\section{CONCLUSION}

In this paper, we presented an adaptive bilateral filter (ABF). The ABF retains the general form of the bilateral filter, but contains two important modifications. First, an offset is $\zeta$ introduced to the range filter in the ABF. Second, both $\zeta$ and the width of the range filter $\sigma_{r}$ in the ABF are locally adaptive. They depend on the local image structure, classified according to the output of the Laplacian of Gaussian (LoG) operator applied to the degraded image. The $\mathrm{ABF}$ outperforms the bilateral filter in noise removal. At the same time, it renders much sharper images than the bilateral filter does.

As a result, the overall quality of the restored image is significantly improved.

\section{REFERENCES}

[1] C. Tomasi and R. Manduchi, "Bilateral filtering forgray and color images," in Proc. Int. Conf. Comput. Vis., 1998, pp. 839-846.

[2] M. Aleksic, M. Smirnov, and S. Goma, "Novel bilateral filter approach: Image noise reduction with sharpening," International Journal of Image Processing and Vision Sciences (IJIPVS), vol. 1, iss. 1, 2012, pp. 60690F1-60690F7.

[3] A. Das and R. M. Rangayyan, "Enhancement of image edge sharpness and acutance," in Proc. SPIE. Soc. Opt. Eng., 1997, vol. 3026, pp. 133-142.

[4] P. Saint-Marc, J. S. Chen, and G. Medioni, "Adaptive smoothing: A general tool for early vision," IEEE Trans. Pattern Analysis and Machine Intelligence, vol. 13, no. 6, p. 514, June 1991.

[5] H. Kotera, Y. Yamada, and K. Shimo, "Sharpness improvement adaptive to edge strength of color image," in Proc. IS\&T/SID Eighth Color Imaging Conf., 2000, pp. 149-154.

[6] S. Fleishman, I. Drori, and D. Cohen-Or, "Bilateral mesh denoising by nonlinear bilateral filtering," ACM Trans. Graph., vol. 22, no. 3, pp. 950-953, 2003, in Proc. SPIE Int. Soc.

[7] S. Yang and K. Hong, "Bilateral interpolation filters for image size Conversion," in Proc. ICIP, 2005, vol. 2, pp. 986-989.

[8] M. Elad, "Retinex by two bilateral filters," in Proc. 5th Int. Conf. ScaleSpace, 2005, pp. 217-229.

[9] S. Paris, P. Kornprobst, J. Tumblin, and F. Durand, "A gentle introduction to bilateral filtering and its applications," presented at the ACMSIGGRAPH, 2007.

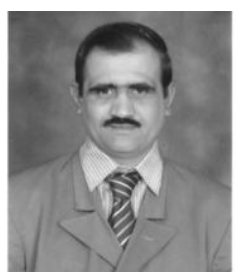

Ch. Ravi Kumar was born at Nellore in Andhra Pradesh in India in 1967. He received B.Sc in physics degree from Nagarjuna University, in 1985, B.E. in electronics from Marathwada University in 1991 and M.E. in electronics with specialization in systems and signal processing from Osmania University in 1998 $\mathrm{He}$ received his doctorate degree from Bharath University Chennai India in 2011 in field of VLSI. From 1992, he is working as an assistant associate and a professor in reputed engineering colleges in A.P. in India. He is currently working as the dean and the head of the Department of Electronics in Prakasam Engineering College, Kandukur, Prakasam (Dt) in A.P in India. He is senior member of IACSIT.

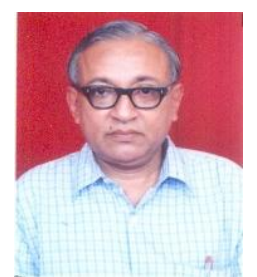

S. K. Srivatsa was born in Bangalore, India on 21st July 1945 . He received his bachelor's degree in electronics and telecommunication engineering from Jadavpur University Calcutta, India and master and $\mathrm{PhD}$ degrees both from Indian Institute of Bangalore, India. He has 37 years of teaching experience. He is a life member/fellow in about 2 dozen registered professional bodies. He has produced $53 \mathrm{PhD}$ 's and he is the author of over 600 publications. He is a recipient of about dozen awards. His field of research interest pertains to electronics and computer science. 